Supporting Information to:

\title{
Structure and Dynamics of L-Selenomethionine in the
}

\section{Solid State.}

Jarosław Gajda, ${ }^{\mathrm{a}}$ Justyna Pacholczyk, ${ }^{\mathrm{a}}$ Anna Bujacz, ${ }^{\mathrm{b}}$ Elżbieta Bartoszak-Adamska, ${ }^{\mathrm{c}}$ Grzegorz Bujacz, ${ }^{\mathrm{b}}$ Włodzimierz Ciesielski, ${ }^{\mathrm{a}}$ and Marek J. Potrzebowski* ${ }^{\text {a }}$

$\mathrm{a}^{*}$ Centre of Molecular and Macromolecular Studies, Polish Academy of Sciences, Sienkiewicza 112 , 90-363 Łódź, Poland. Fax: +48 42680 3261; Tel: +48 42680 3240;

${ }^{\mathrm{b}}$ Institute of Technical Biochemistry, Technical University of Łódź, Stefanowskiego 4/10, 90-924 Łódź, Poland. Fax: +48 42631 3402; Tel:+ 4842631 3431;

${ }^{\mathrm{c}}$ Adam Mickiewicz University, Department of Chemistry, Grunwaldzka 6, 60-780 Poznań, Poland. Fax: +48 61829 1273; Tel: +48 61829 1273;

Corresponding author: Marek Potrzebowski, Centre of Molecular and Macromolecular Studies, Polish Academy of Sciences, Sienkiewicza 112, 90-363 Łódź, Poland. Fax: +48 42680 3261; Tel: +48 42680 3240; E-mail: marekpot@,bilbo.cbmm.lodz.pl 


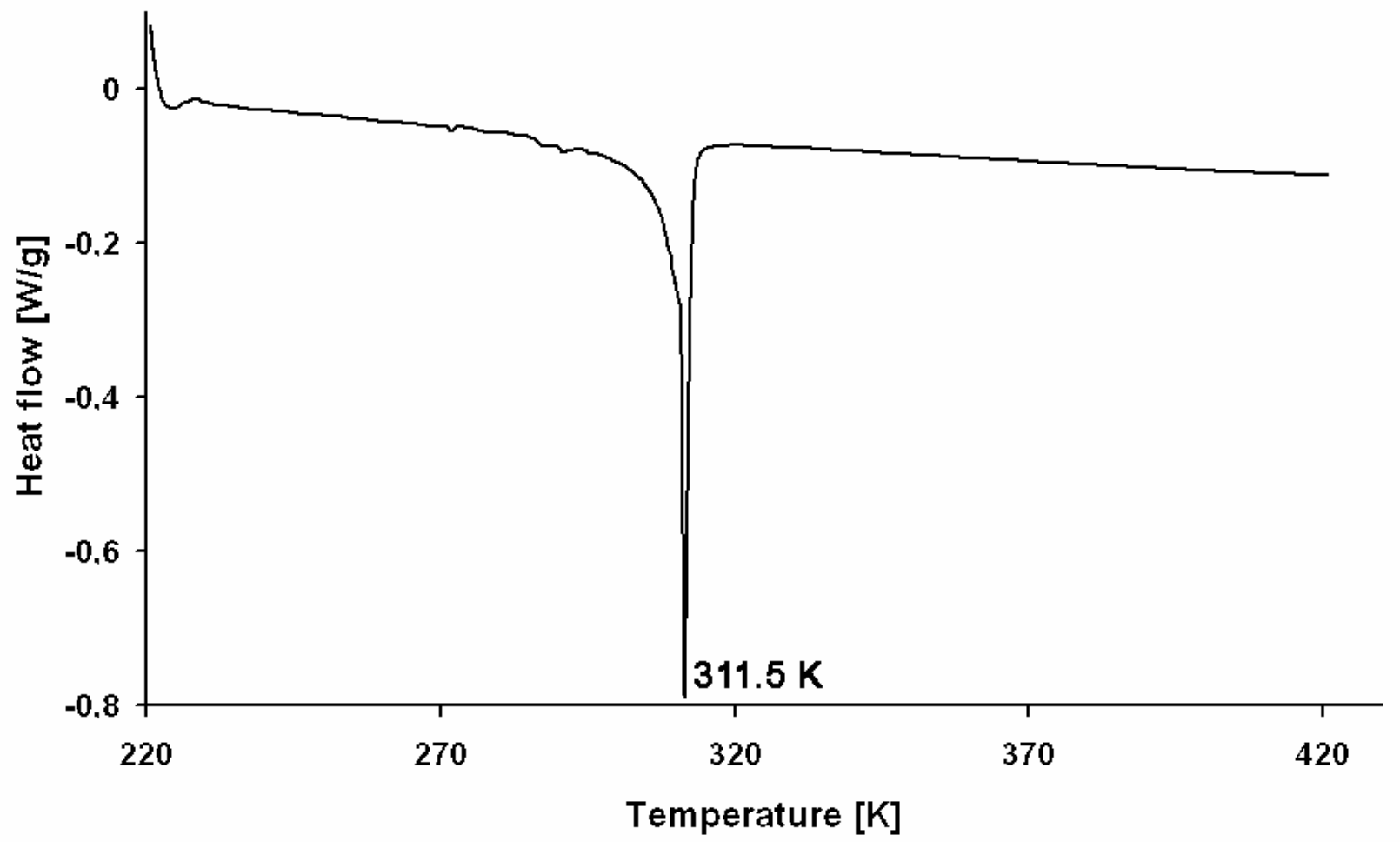

Figure S2. DSC profile of L-selenomethionine. 
a)
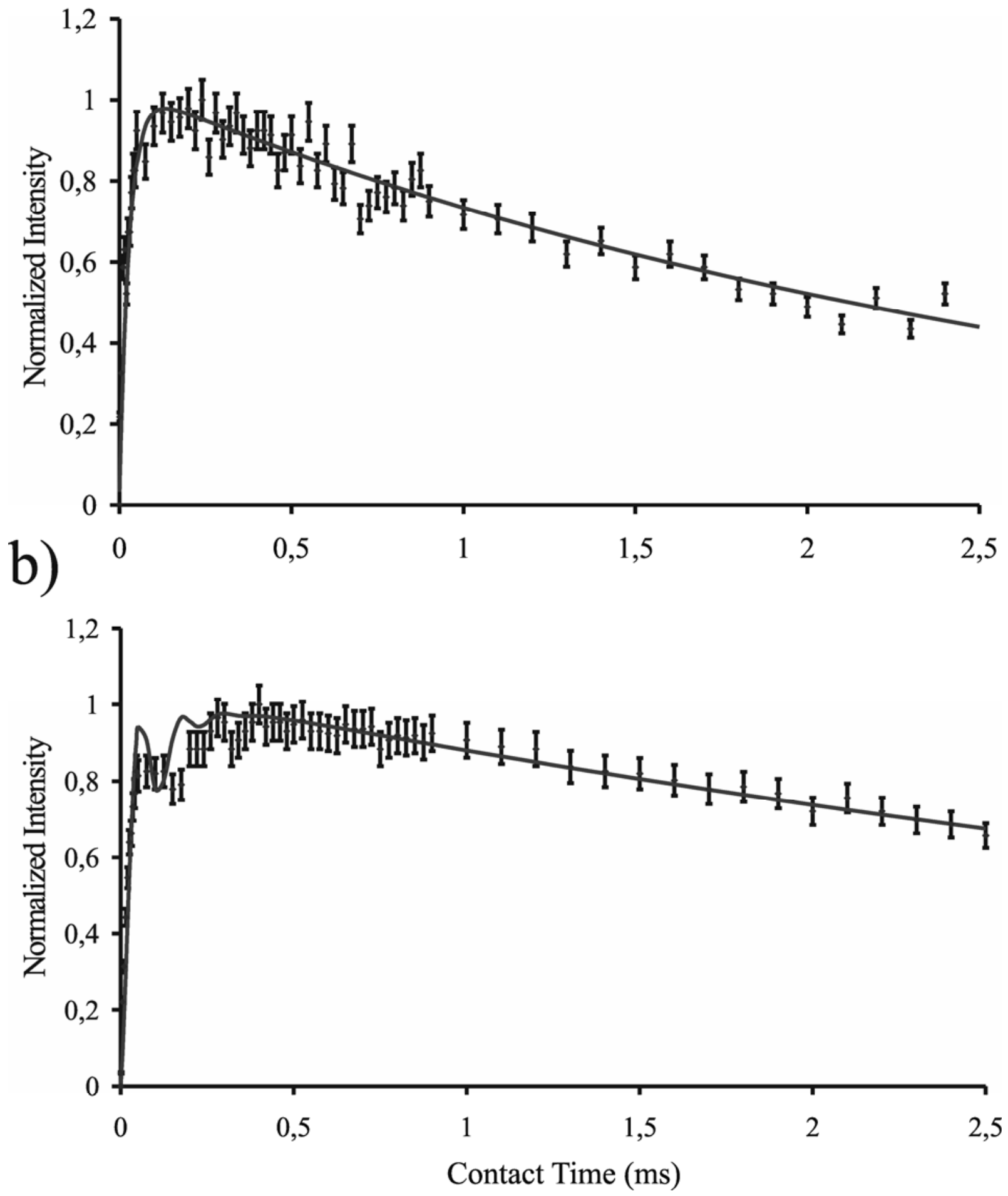

Figure $\mathrm{S} 3$. The $\mathrm{CP}$ profiles for $\mathrm{C}_{\beta}$ carbons of molecule B a) $193 \mathrm{~K}$ and b) $293 \mathrm{~K}$ as a function of contact time (a) fitting using I-I*-S model: $T_{1 \rho H}=4,05 \mathrm{~ms}, \lambda=0,17, \mathrm{~T}_{2}=60 \mu \mathrm{s} \mathrm{T}_{\mathrm{df}}=150 \mu \mathrm{s}$ (b) $T_{1 \rho H}=5,65 \mathrm{~ms}$, $\mathrm{b}=12,5 \mathrm{kHz}, \mathrm{T}_{\mathrm{df}}=100 \mu \mathrm{s}$. Experimental points with $5 \%$ errors marked. 


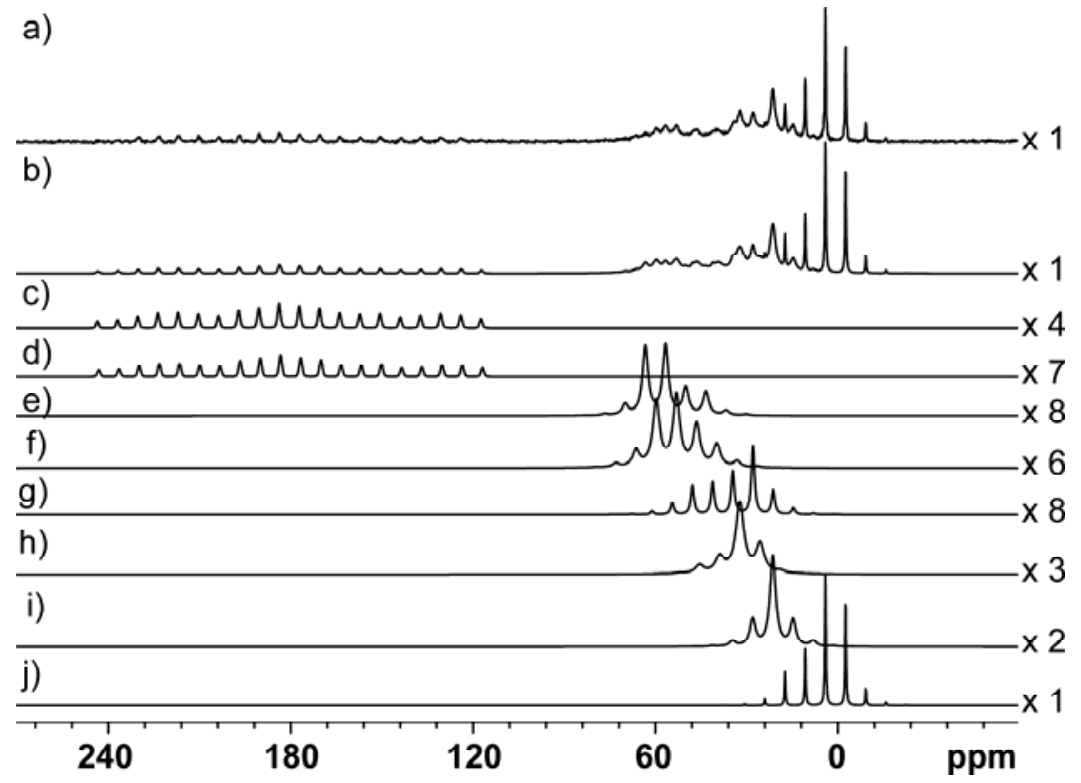

Figure S4. ${ }^{13} \mathrm{C}$ MAS spectrum of L-selenomethionine recorded at spinning rate of $500 \mathrm{~Hz}$ at 278 (a), simulated spectrum (b) with separated spinning sideband systems: c - $C_{0}(B), d-C_{0}(A), e-C_{\alpha}(A), f-$ $\mathrm{C}_{\alpha}(\mathrm{B}), \mathrm{g}-\mathrm{C}_{\beta}(\mathrm{A}), \mathrm{h}-\mathrm{C}_{\beta}(\mathrm{B}), \mathrm{i}-\mathrm{C}_{\gamma} \mathrm{j}-\mathrm{C}_{\delta}$. The subspectra are shown with appropriate scaling factors (right columns) in order to better visualize the spinning sideband pattern. 


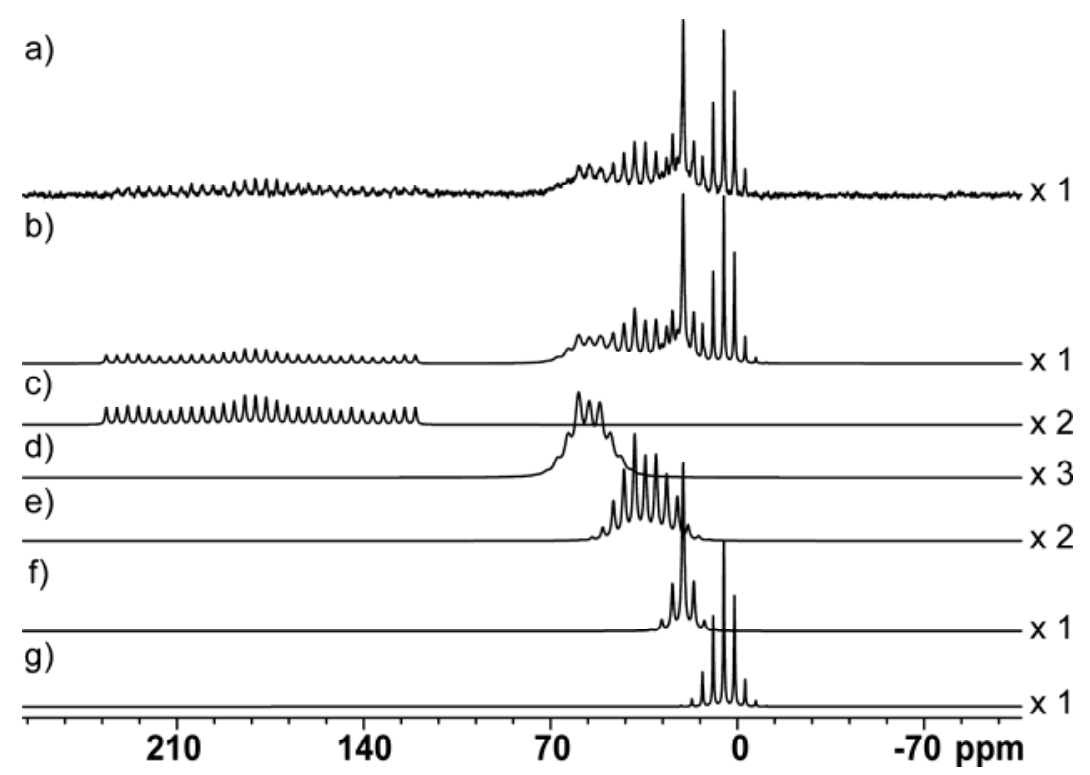

Figure S5. ${ }^{13} \mathrm{C}$ MAS spectrum of L-selenomethionine recorded at spinning rate of $300 \mathrm{~Hz}$ at $313 \mathrm{~K}$ (a), simulated spectrum (b), with separated spinning sideband systems: $\mathrm{c}-\mathrm{C}_{0}, \mathrm{~d}-\mathrm{C}_{\alpha}, \mathrm{e}-\mathrm{C}_{\beta}, \mathrm{f}-\mathrm{C}_{\gamma}, \mathrm{g}-\mathrm{C}_{\delta}$. The subspectra are shown with appropriate scaling factors (right columns) in order to better visualize the spinning sideband pattern. 
a)

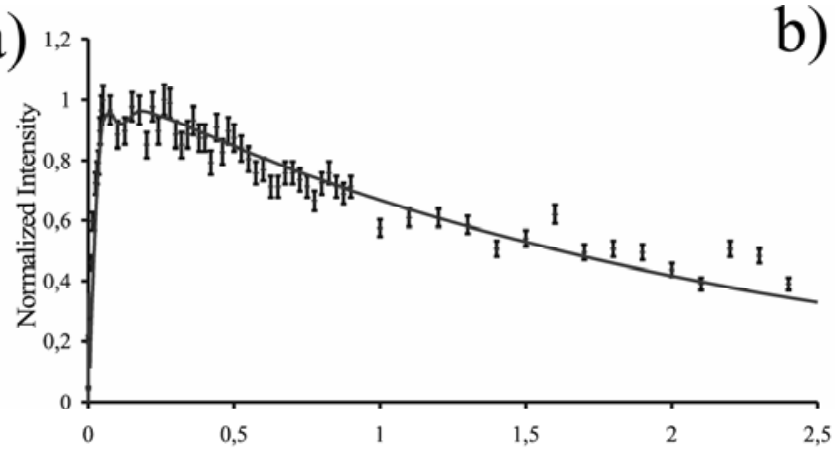

b)

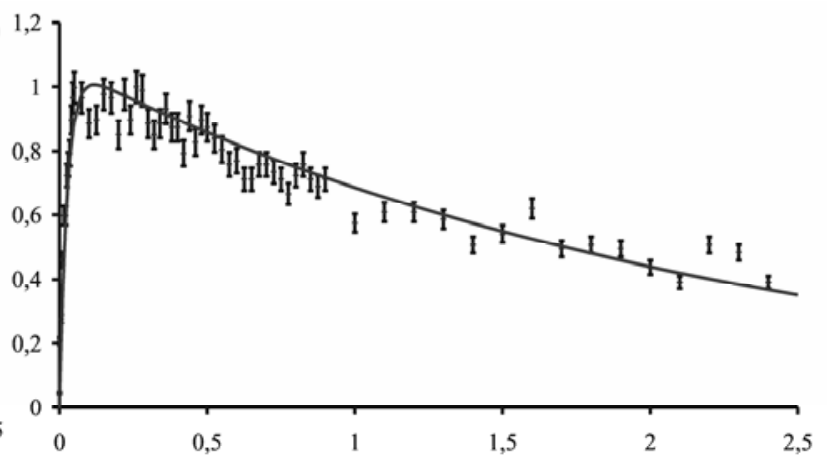

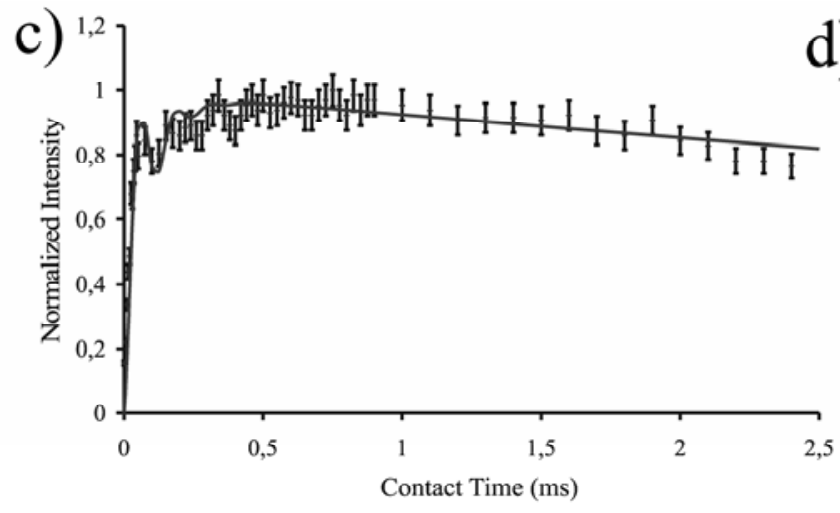

d)

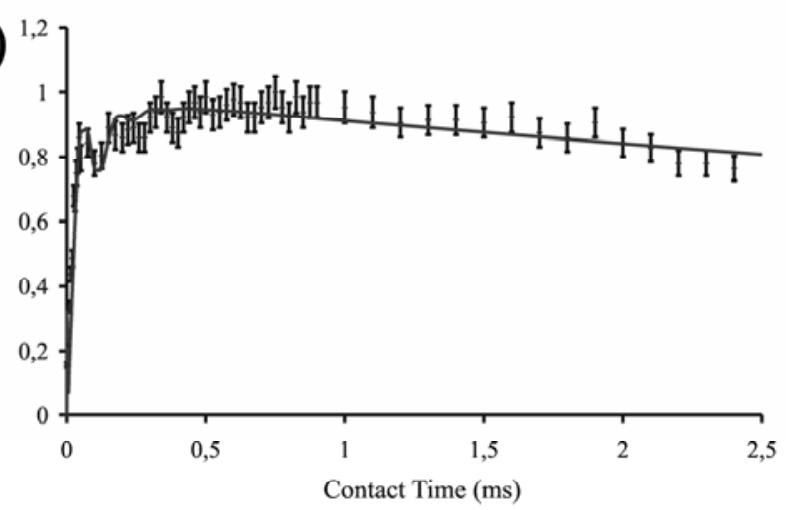

Figure S6. The CP profiles for $\mathrm{C}_{\alpha}$ atoms at $293 \mathrm{~K}$ as a function of contact time (a) molecule A signal fitted using equation $1\left(T_{1 \rho H}=2,11 \mathrm{~ms}, \mathrm{~b}=10,1 \mathrm{kHz}, \mathrm{T}_{\mathrm{df}}=60 \mu \mathrm{s}\right)$; (b) molecule A using equation 2 $\left(T_{1 \rho H}=2,11 \mathrm{~ms}, \lambda=0,18, \mathrm{~T}_{2}=100 \mu \mathrm{s}, \mathrm{T}_{\mathrm{df}}=40 \mu \mathrm{s}\right)$. (c) molecule $\mathrm{B}$ signal fitted using equation 1 $\left(T_{1 \rho H}=12,5 \mathrm{~ms}, \mathrm{~b}=15,0 \mathrm{kHz}, \mathrm{T}_{\mathrm{df}}=99 \mu \mathrm{s}\right) ;(\mathrm{d})$ molecule B using equation $2\left(T_{1 \rho H}=12,5 \mathrm{~ms}, \lambda=0,11\right.$, $\left.\mathrm{T}_{2}=99 \mu \mathrm{s}, \mathrm{T}_{\mathrm{df}}=108 \mu \mathrm{s}\right)$. Experimental points with $5 \%$ errors marked. 
Table $\mathrm{S} 1 .{ }^{13} \mathrm{C} \mathrm{T}_{1}$ relaxation times in temperature range $193 \mathrm{~K}-293 \mathrm{~K}$

\begin{tabular}{lllllll}
\hline \multicolumn{7}{c}{${ }^{13} \mathrm{C}_{1}(\mathrm{~s})$} \\
\hline $\mathrm{T}[\mathrm{K}\}$ & $\mathrm{C}_{\alpha}(\mathrm{A})$ & $\mathrm{C}_{\alpha}(\mathrm{B})$ & $\mathrm{C}_{\beta}(\mathrm{A})$ & $\mathrm{C}_{\beta}(\mathrm{B})$ & $\mathrm{C}_{\gamma}$ & $\mathrm{C}_{\delta}$ \\
193 & 204.00 & 126.00 & 158.00 & 33.00 & 68.00 & 1.77 \\
213 & 73.50 & 36.90 & 42.90 & 16.52 & 12.80 & 2.34 \\
233 & 61.70 & 17.66 & 26.24 & 13.81 & 12.34 & 2.25 \\
253 & 40.65 & 13.04 & 19.96 & 6.15 & 11.70 & 2.36 \\
273 & 42.19 & 8.18 & 15.12 & 3.30 & 6.89 & 3.03 \\
293 & 38.24 & 5.35 & 10.77 & 2.12 & 4.05 & 4.23 \\
\hline
\end{tabular}

